\title{
A Multi-Resolution Framework for Diffusion Tensor Images
}

\author{
Luc Florack \\ Department of Mathematics and Computer Science \& Department of Biomedical Engineering \\ Eindhoven University of Technology, PO Box 513, 5600 MB Eindhoven, The Netherlands \\ Laura Astola \\ Department of Mathematics and Computer Science \\ Eindhoven University of Technology, PO Box 513, 5600 MB Eindhoven, The Netherlands \\ \{L.M.J.Florack, L.J.Astola\}etue.nl
}

\begin{abstract}
A new scale space paradigm is proposed for multiresolution analysis of diffusion tensor images (DTI). An a priori consistency requirement is stipulated, which precludes a linear model. A nonlinear adaptation is proposed to remedy the problem. Subsequently it is shown how differentiation can be operationalized.

Considerations in this paper are relevant for DTI analysis in a differential geometric framework, in which the DTI image imposes a Riemannian structure. As such it adds further support in favor of the "geometric rationale", opening the door for a multi-resolution geometric approach towards fibre tracking, connectivity analysis, and so forth.
\end{abstract}

Keywords. Scale space, diffusion tensor imaging, Riemannian geometry, log-Euclidean framework, differentiation.

\section{Introduction}

Recent literature advocates differential geometry ${ }^{1}$ as a powerful mathematical framework for analyzing diffusion tensor images (DTI) [2, 8, 23, 34, 36]. However, operationalization of differential concepts is hampered by the ill-posed nature of classical differential operators. This has led to the introduction of regularization techniques [30, 34, 40], not only in DTI, but in image analysis in general.

An intrinsic coupling of regularization and differentiation

${ }^{1}$ Geometric considerations have been applied to domain and codomain of a DTI image. Our focus is the former but on the fly we obtain a natural connection to the latter. is achieved by treating images as tempered distributions [37]. In the framework of distribution theory the act of differentiation (including zeroth order) requires a class of smooth test functions (linear filters), by virtue of which it becomes well-posed. In scale space theory the class of test functions is axiomatically restricted so as to arrive at an operational definition of an image derivative $[7,9,10,15,17,18,19,20,21,22,24,31,38,44,45]$. The paradigmatic (zeroth order) filter is the normalized Gaussian of a priori arbitrary scale. This naturally produces a scale space representation, i.e. a continuous family of images intended to capture raw image data at arbitrary levels of resolution (within physically reasonable limits). This holds a fortiori for any image derivative.

Linear scale space theory provides a natural framework for image differentiation in the absence of constraints that are incompatible with linearity. An instance of incompatibility is encountered in image processing by nonlinear diffusion $[16,35,42,43]$, in which the desire to "preserve edges" precludes linearity. Another instance in which linearity conflicts with fundamental demands - and the subject of this paper-is encountered in the context of DTI.

A DTI sample is a symmetric positive definite matrix (more precisely, a contravariant 2-tensor [26, 39]). In the differential geometric rationale its inverse defines the components of the Riemannian metric tensor. The first order structure of the DTI image then induces a unique, so-called affine connection, which is the geometric construct for "fitting together" neighbouring tangent spaces, allowing e.g. fibre tracking via parallel transport (geodesics). In turn, the first order structure of this connection determines the Riemann curvature tensor ${ }^{2}$, which can be seen as a measure of geodesic deviation (inclination of neighbouring fibres to

\footnotetext{
${ }^{2}$ In $3 \mathrm{D}$ the Riemann tensor is equivalent to the so-called Ricci tensor.
} 
repel or attract eachother [2]) in analogy with the relative acceleration experienced by freely falling particles in an inhomogeneous gravitational field [26].

In view of the above, we propose a multi-resolution framework for DTI images, so as to admit well-posed differentiation at any level of resolution. The space of symmetric positive definite matrices is closed under inversion. A DTI image and its pointwise inverse are dual metrics, and their matrix representations belong to the same class of positive definite symmetric matrix fields. A scale space generator for this class must therefore be such that it manifestly preserves inverse relationships at all scales. In other words, the acts of blurring and inversion of matrix fields should commute. One easily verifies that this a priori commutativity requirement is inconsistent with linear blurring.

After establishing some notation in the next section we address the problem of how to set up a consistent scale space paradigm for DTI in Section 3. In Section 4 we operationalize differential structure, and we conclude in Section 5.

\section{Notation}

We denote a DTI-image by $f: \mathbb{R}^{n} \rightarrow \mathbb{S}_{n}^{+}$, where $\mathbb{S}_{n}^{+} \subset$ $\mathbb{S}_{n} \subset \mathbb{M}_{n}$ denotes the set of $\mathbb{R}$-valued symmetric positive definite $n \times n$ matrices ${ }^{3}, \mathbb{S}_{n}$ the set of $\mathbb{R}$-valued symmetric $n \times n$ matrices, and $\mathbb{M}_{n}$ the set of all $n \times n$ matrices. Its pointwise inverse is $f^{\text {inv }}: \mathbb{R}^{n} \rightarrow \mathbb{S}_{n}^{+}$, so that $\left(f^{\text {inv }} f\right)(x)=$ $\left(f f^{\text {inv }}\right)(x)=I$, the identity matrix, at each point $x \in \mathbb{R}^{n}$.

$C^{\omega}\left(\mathbb{R}^{n}, \mathbb{M}_{n}\right)$ denotes the class of analytical functions $f: \mathbb{R}^{n} \rightarrow \mathbb{M}_{n}$. Self-explanatory definitions hold for $C^{\omega}\left(\mathbb{R}^{n}, \mathbb{S}_{n}^{+}\right) \subset C^{\omega}\left(\mathbb{R}^{n}, \mathbb{S}_{n}\right) \subset C^{\omega}\left(\mathbb{R}^{n}, \mathbb{M}_{n}\right)$.

The isotropic Gaussian scale space kernel in $n$ dimensions is given by

$$
\phi_{\sigma}(x)=\frac{1}{{\sqrt{2 \pi \sigma^{2}}}^{n}} \exp \left(-\frac{1}{2} \frac{\|x\|^{2}}{\sigma^{2}}\right) .
$$

The scale space representation of $f \in C^{\omega}\left(\mathbb{R}^{n}, \mathbb{S}_{n}^{+}\right)$is obtained by the blurring operator (detailed in the next section)

$$
\mathscr{F}: C^{\omega}\left(\mathbb{R}^{n}, \mathbb{S}_{n}^{+}\right) \times \mathbb{R}^{+} \rightarrow C^{\omega}\left(\mathbb{R}^{n}, \mathbb{S}_{n}^{+}\right):(f, \sigma) \mapsto \mathscr{F}(f, \sigma)
$$

We shall require $\mathscr{F}(f, 0)=f$ for all $f \in C^{\omega}\left(\mathbb{R}^{n}, \mathbb{S}_{n}^{+}\right)$, and sometimes use the shorthand notation ${ }^{4} f_{\sigma} \equiv \mathscr{F}(f, \sigma)$.

\footnotetext{
${ }^{3}$ In practice $n=3$. For the sake of illustration we also consider $n=2$.

${ }^{4}$ Analyticity of $\mathscr{F}(f, 0) \in C^{\omega}\left(\mathbb{R}^{n}, \mathbb{S}_{n}^{+}\right)$is not essential.
}

\section{The Basic Paradigm}

\subsection{Formulation of Consistency}

Our goal is to formulate a consistent scale space representation for symmetric positive definite matrix fields $f \in C^{\omega}\left(\mathbb{R}^{n}, \mathbb{S}_{n}^{+}\right)$. Since inversion is automorphic on $C^{\omega}\left(\mathbb{R}^{n}, \mathbb{S}_{n}^{+}\right)$, there is no mathematical justification for treating $f$ and $f^{\text {inv }}$ differently. Thus consistency means that if $f_{\sigma}=\mathscr{F}(f, \sigma)$, then for each $\sigma \in \mathbb{R}^{+}$we must have $f_{\sigma}^{\text {inv }}=\mathscr{F}\left(f^{\text {inv }}, \sigma\right)$, i.e. blurring and inversion must commute. Note that commutativity would not hold if we would define $\mathscr{F}(f, \sigma)=f * \phi_{\sigma}$, since $f^{\text {inv }} * \phi_{\sigma} \neq\left(f * \phi_{\sigma}\right)^{\text {inv }}$ for $\sigma>0$. Consistency requires the scale space generator $\mathscr{F}$ to be nonlinear. In the spirit of previous work $[4,11]$ —applicable to scalar images-we will look for a pseudo-linear scale space representation with the desired commutativity property. We start with an intermezzo on relevant results in the context of matrix-valued functions.

\subsection{Matrix Exponential and Logarithm}

The exponential map exp : $\mathbb{M}_{n} \rightarrow \mathrm{GL}_{n}$ maps an arbitrary $n \times n$ matrix to a nonsingular matrix, i.e. an element of the general linear group $[12,13,27]$. For later convenience we define $\mathbb{M}_{n}^{+}=\exp \left(\mathbb{M}_{n}\right) \subset \mathrm{GL}_{n}$. For our purpose it suffices to consider diagonalizable matrices. In fact, we need only consider elements of $\mathbb{S}_{n} \subset \mathbb{M}_{n}$, which are diagonalizable with real eigenvalues, in which case the range of the exponential map equals $\exp \left(\mathbb{S}_{n}\right)=\mathbb{S}_{n}^{+}$. So we will employ the prototype

$$
\exp : \mathbb{S}_{n} \rightarrow \mathbb{S}_{n}^{+}: A \mapsto \exp A
$$

An operational representation of a general analytical matrix function is given by Sylvester's formula ${ }^{5}$ :

$$
F(A) \stackrel{\text { def }}{=} \sum_{i=1}^{m} F\left(\lambda_{i}\right) A_{i},
$$

in which the $\lambda_{i}, i=1, \ldots m$, are all distinct eigenvalues of $A$. In Eq. (4) the left hand side-with intentional abuse of notation-is defined by virtue of the analytical scalar function $F \in C^{\omega}(\mathbb{R}, \mathbb{R})$ on the right hand side, i.c. $F \equiv \exp$, and the so-called Frobenius covariants are given by

$$
A_{i}=\prod_{j=1, j \neq i}^{m} \frac{1}{\lambda_{i}-\lambda_{j}}\left(A-\lambda_{j} I\right)
$$

\footnotetext{
${ }^{5}$ Generically one expects $m=n$ a.e. within the DTI image domain.
} 
The logarithmic map, restricted to $\mathbb{S}_{n}^{+}$for our purposes, has prototype

$$
\ln : \mathbb{S}_{n}^{+} \rightarrow \mathbb{S}_{n}: B \mapsto \ln B,
$$

and is the unique inverse of the exponential map restricted to $\mathbb{S}_{n}: \ln \left(\mathbb{S}_{n}^{+}\right)=\mathbb{S}_{n}$. In this case we may apply Eqs. (4-5) with $F \equiv \ln$.

\subsection{Multi-Scale Representation}

We shall be primarily interested in a second order multiscale representation. This suffices for nearly all applications that exploit differential geometry, such as the computation of Christoffel symbols [23, 34, 36] and the Riemann curvature tensor [2] compatible with the metric. Generalization to higher orders is straightforward.

Let us start by considering the basic zeroth order scale space paradigm, accounting for commutativity of blurring and inversion. Subsequently we turn to first and second order differential structure of the established representation.

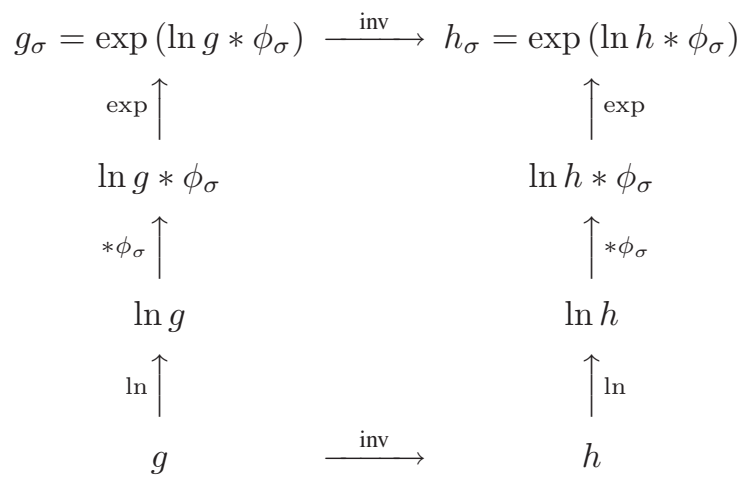

Figure 1. Commuting diagram for blurring and inversion.

Fig. 1 shows how a consistent multi-scale representation can be obtained. Indeed, if $f \in C^{\omega}\left(\mathbb{R}^{n}, \mathbb{S}_{n}^{+}\right)$, then $f_{\sigma}=$ $\mathcal{F}(f, \sigma)$ constructed according to

$$
\mathcal{F}(f, \sigma)=\exp \left(\ln f * \phi_{\sigma}\right),
$$

satisfies the desired commutativity property,

$$
\mathcal{F}\left(f^{\text {inv }}, \sigma\right)=\mathcal{F}(f, \sigma)^{\text {inv }} .
$$

This follows immediately by inspection of Fig. 1 and Eq. (7), using the identities

$$
\exp (-A)=(\exp A)^{\mathrm{inv}} \quad \text { and } \quad \ln B^{\mathrm{inv}}=-\ln B,
$$

for $A \in \mathbb{S}_{n}, B \in \mathbb{S}_{n}^{+}$. The zeroth order scheme, Eq. (7), is in line with the so-called log-Euclidean framework proposed by Arsigny et al., Pennec et al., and Fillard et al. [1, 8, 34], but is here based on a different motivation, viz. the fundamental commutativity axiom. In this sense the zeroth order result comes out with no surprise. However, the differential geometric rationale also compels us to consider derivatives of this zeroth order multi-scale representation. In the next section we show that this is not quite trivial, but that closedform expressions can be obtained in this respect as well.

\section{Differentiation}

A complication arises when differentiating the zeroth order representation, Eq. (7), due to the fact that matrices do not in general commute. In particular this complicates the chain rule. To appreciate this, we recall a few nontrivial results on derivatives of a matrix exponential function. (These results are formulated for arbitrary, sufficiently regular $n \times n$ matrix fields $[3,5,13,28,29,32]$.)

Definition 1 Let $X \in C^{\omega}\left(\mathbb{R}, \mathbb{M}_{n}\right)$. The parametric derivative of the matrix exponential function $\exp X \in$ $C^{\omega}\left(\mathbb{R}, \mathbb{M}_{n}^{+}\right)$is defined as

$\frac{d}{d t} \exp (X(t))=\lim _{h \rightarrow 0} \frac{\exp (X(t)+h \dot{X}(t))-\exp (X(t))}{h}$.

Theorem 1 The parametric derivative of a one-parameter matrix-valued function $X \in C^{\omega}\left(\mathbb{R}, \mathbb{M}_{n}\right)$ can be operationalized as follows:

$\frac{d}{d t} \exp (X(t))=\int_{0}^{1} \exp ((1-\alpha) X(t)) \frac{d X}{d t}(t) \exp (\alpha X(t)) d \alpha$.

In order to prove this the following lemma turns out useful, the proof of which follows immediately by substitution.

Lemma 1 The unique solution of the inhomogeneous matrix-ODE

$$
\frac{d Y}{d \lambda}=X Y+F \quad \text { with initial condition } Y(0)=Y_{0},
$$

with $X \in \mathbb{M}_{n}$ constant, and $F \in C^{\omega}\left(\mathbb{R}, \mathbb{M}_{n}\right)$, is given by

$$
Y(\lambda)=\exp (\lambda X)\left(Y_{0}+\int_{0}^{\lambda} \exp (-\mu X) F(\mu) d \mu\right) \text {. }
$$

Proof of Theorem 1. Consider the following matrix-ODE, with $X, V \in \mathbb{M}_{n}$ constant, and $h \in \mathbb{R}$ :

$$
\frac{d Y}{d \lambda}=(X+h V) Y \quad \text { with initial condition } Y(0)=I \text {. }
$$


The solution-obtained by identifying the term $h V Y$ with the inhomogeneous term $F$ in Lemma 1, with $Y_{0}=I$-can be represented in the form of a Volterra integral equation:

$$
Y(\lambda)=\exp (\lambda X)+h \int_{0}^{\lambda} \exp ((\lambda-\alpha) X) V Y(\alpha) d \alpha .
$$

This gives rise to an $\mathcal{O}(h)$ approximation

$$
Y(\lambda)=\exp (\lambda X)+h \int_{0}^{\lambda} \exp ((\lambda-\alpha) X) V \exp (\alpha X) d \alpha+\mathcal{O}\left(h^{2}\right) .
$$

On the other hand we also have the exact solution,

$$
Y(\lambda)=\exp (\lambda(X+h V)) .
$$

Identifying the last two expressions, putting $\lambda=1$, yields

$$
\begin{aligned}
& \exp (X+h V)-\exp (X)= \\
& \quad h \int_{0}^{1} \exp ((1-\alpha) X) V \exp (\alpha X) d \alpha+\mathcal{O}\left(h^{2}\right),
\end{aligned}
$$

from which the proof readily follows.

We define the gradient of a multivariate matrix-valued function $X \in C^{\omega}\left(\mathbb{R}^{k}, \mathbb{M}_{n}\right), \nabla \exp (X)$, in a similar fashion. We may generalize Theorem 1 accordingly.

Theorem 2 Let $X \in C^{\omega}\left(\mathbb{R}^{k}, \mathbb{M}_{n}\right)$. The gradient of the matrix exponential function $\exp X \in C^{\omega}\left(\mathbb{R}^{k}, \mathbb{M}_{n}^{+}\right)$can be represented component-wise as follows:

$\partial_{\mu} \exp (X(x))=\int_{0}^{1} \exp ((1-\alpha) X(x)) \partial_{\mu} X(x) \exp (\alpha X(x)) d \alpha$.

Proof of Theorem 2. Freeze all arguments except $x^{\mu}$, and apply Theorem 1 .

By repetitive application of Theorem 2, using the product rule, we obtain higher order derivatives. (The product rule readily generalizes to matrix-valued functions, as long as one respects the ordering of non-commuting matrices.) We refer to Appendix A for the second order case, presenting the operational formula for the Hessian, $\nabla^{2} \exp (X)$. It is clear that complexity increases rapidly with order.

In the context of DTI we set $k=n$, and restrict ourselves to $\mathbb{S}_{n} \subset \mathbb{M}_{n}, \mathbb{S}_{n}^{+} \subset \mathbb{M}_{n}^{+}$. In the following, logarithm takes precedence over convolution product:

Result 1 Setting $X=\ln f * \phi_{\sigma}$, we have obtained formulas for the gradient-Theorem 2-and Hessian-Theorem 3, Appendix $A-$ of the multi-scale representation of a matrix field $f \in C^{\omega}\left(\mathbb{R}^{n}, \mathbb{S}_{n}^{+}\right)$.
Moreover, if $X=\ln f * \phi_{\sigma}$, then $\partial_{\mu} X=\ln f * \partial_{\mu} \phi_{\sigma}$, $\partial_{\mu} \partial_{\nu} X=\ln f * \partial_{\mu} \partial_{\nu} \phi_{\sigma}$. These are the familiar expressions for derivatives in linear scale space theory, applied to the logarithm of the input image, and can be computed in the same way (by direct convolution, via DFT, or recursively $[6,14,17,41])$. However, to obtain the desired multi-scale derivatives requires us to compute matrix logarithms and exponentials, and to compute the aforementioned integral representations (Theorems 2-3) over non-commuting matrix products, e.g. using a standard numerical Riemann sum approximation for the parameter integrals $[3,5]$. Although technically straightforward, this is a numerical complication, but it is the price we must pay for consistency. Up to second order, the relevant case in the differential geometric rationale, this fortunately turns out relatively unproblematic. Figs. 2-3 illustrate the zeroth order scale space representation of a synthetic tensor field $f \in C^{\omega}\left(\mathbb{R}^{2}, \mathbb{S}_{2}^{+}\right)$. (Higher order results are difficult to visualize, and best studied in the context of a particular application, such as tractography or connectivity analysis.) Asymptotics are as expected. Given suitable boundary conditions, the blurred DTI matrix field tends to an isotropic, homogeneous field given by the pointwise identity matrix as scale tends to infinity. The smooth connection between Euclidean geometry at large scales and the Riemannian geometry induced by the raw DTI image at data scale opens the door to a coarse-tofine approach in differential geometric analysis.

\section{Conclusion and Summary}

We have proposed an operational scale space paradigm for symmetric positive definite matrix fields, such as encountered in DTI. The automorphic nature of matrix inversion within this class led us to impose a basic consistency demand, viz. that blurring and inversion should commute. This demand is incompatible with linear scale space theory, but is manifest in the proposed nonlinear modification. Perhaps not surprisingly, the commutativity axiom reproduces the zeroth order representation introduced by Arsigny et al., Fillard et al., and Pennec et al. As such it adds further support to their so-called log-Euclidean framework [1, 8, 34].

A complication is the nontrivial nature of the chain rule for nested matrix-valued functions. We have derived novel, explicit integral expressions for first and second order derivatives, and provided an operational scheme to compute these at any physically meaningful scale. Extension to higher orders is straightforward, but produces cumbersome expressions, with a concomitant computational price due to the fact that the complexity of the resulting integral expressions grows rapidly with order. We have argued, however, that second order structure may well be sufficient in a differen- 
tial geometric framework, since it allows one to obtain the most important geometric objects, notably the affine connection or Christoffel symbols, and the Riemann curvature tensor. Numerical implementation is straightforward.

Interestingly, the proposed multi-resolution scheme naturally joins the differential geometric paradigms on the DTI domain (Riemannian geometry) and codomain (logEuclidean framework). An intriguing, hitherto unexplored possibility enabled by this scheme is a coarse-tofine (or Euclidean-to-Riemannian) approach towards differential geometric DTI analysis (tractography, connectivity analysis, and so forth). In this respect one should always keep in mind that resolution limitations in diffusion imaging preclude a microscopic analysis of biological tissue at cellular level, so that data scale is a priori as arbitrary as any other empirical scale. One is thus confined to a mesoscopic analysis, in which tissue organization induces the "right scales" for analysis rather than technical acquisition limitations (pixels). In a multi-scale representation all scales are a priori equivalent, thus providing a natural basis for probing the "deep structure" of DTI data [21]. This is a subject for much future work.

The proposed multi-resolution framework is also relevant for high angular resolution diffusion imaging (HARDI), since in this context, too, the differential geometric rationale is applicable, $\mathrm{cf}$. the active contour approach based on Finsler geometry by Melonakos et al. [25], and the intimate analytical connection between HARDI and DTI pointed out by Özarslan and Mareci [33].

\section{Acknowledgements.}

The Netherlands Organisation for Scientific Research (NWO) is gratefully acknowledged for financial support.

\section{References}

[1] V. Arsigny, P. Fillard, X. Pennec, and N. Ayache. LogEuclidean metrics for fast and simple calculus on diffusion tensors. Magnetic Resonance in Medicine, 56(2):411-421, 2006.

[2] L. Astola, L. Florack, and B. ter Haar Romeny. Measures for pathway analysis in brain white matter using diffusion tensor images. In N. Karssemeijer and B. Lelieveldt, editors, Proceedings of the Fourteenth International Conference on Information Processing in Medical ImagingIPMI 2007 (Kerkrade, The Netherlands), volume 4584 of Lecture Notes in Computer Science, pages 642-649, Berlin, 2007. Springer-Verlag.
[3] R. W. Atherton and A. E. de Gance. On evaluation of the derivative of the matrix exponential function. IEEE Transactions on Automatic Control, 20(5):707-708, October 1975.

[4] B. Burgeth and J. Weickert. An explanation for the logarithmic connection between linear and morphological systems. In Griffin and Lillholm [15], pages 325-339.

[5] E. A. De Souza Neto. The exact derivative of the exponential of an unsymmetric tensor. Computer Methods in Applied Mechanics and Engineering, 190:2377-2383, 2001.

[6] R. Deriche. Recursively implementing the Gaussian and its derivatives. In V. Srinivasan, O. Sim Heng, and A. Yew Hock, editors, Proceedings of the 2nd Singapore International Conference on Image Processing (Singapore, September 1992), pages 263-267. World Scientific, Singapore, 1992.

[7] R. Duits, L. Florack, J. de Graaf, and B. ter Haar Romeny. On the axioms of scale space theory. Journal of Mathematical Imaging and Vision, 20(3):267-298, 2004.

[8] P. Fillard, X. Pennec, V. Arsigny, and N. Ayache. Clinical DT-MRI estimation, smoothing, and fiber tracking with logEuclidean metrics. IEEE Transactions on Medical Imaging, 26(11), November 2007.

[9] L. M. J. Florack. Image Structure, volume 10 of Computational Imaging and Vision Series. Kluwer Academic Publishers, Dordrecht, The Netherlands, 1997.

[10] L. M. J. Florack, B. M. t. Haar Romeny, J. J. Koenderink, and M. A. Viergever. Linear scale-space. Journal of Mathematical Imaging and Vision, 4(4):325-351, 1994.

[11] L. M. J. Florack, R. Maas, and W. J. Niessen. Pseudo-linear scale-space theory. International Journal of Computer Vision, 31(2/3):247-259, April 1999.

[12] T. C. Fung. Computation of the matrix exponential and its derivatives by scaling and squaring. International Journal for Numerical Methods in Engineering, 59:1273-1286, 2004.

[13] F. R. Gantmacher. The Theory of Matrices. American Mathematical Society, 2001.

[14] J.-M. Geusebroek, A. W. M. Smeulders, and J. van de Weijer. Fast anisotropic Gauss filtering. IEEE Transactions on Image Processing, 12(8):938-943, 2003.

[15] L. D. Griffin and M. Lillholm, editors. Scale-Space Methods in Computer Vision: Proceedings of the Fourth International Conference, Scale-Space 2003, Isle of Skye, UK, volume 2695 of Lecture Notes in Computer Science. SpringerVerlag, Berlin, June 2003.

[16] B. M. t. Haar Romeny, editor. Geometry-Driven Diffusion in Computer Vision, volume 1 of Computational Imaging and Vision Series. Kluwer Academic Publishers, Dordrecht, 1994.

[17] B. M. t. Haar Romeny. Front-End Vision and Multi-Scale Image Analysis: Multi-Scale Computer Vision Theory and Applications, written in Mathematica, volume 27 of Computational Imaging and Vision Series. Kluwer Academic Publishers, Dordrecht, The Netherlands, 2003.

[18] B. M. t. Haar Romeny, L. M. J. Florack, J. J. Koenderink, and M. A. Viergever, editors. Scale-Space Theory in Computer Vision: Proceedings of the First International Conference, 
Scale-Space'97, Utrecht, The Netherlands, volume 1252 of Lecture Notes in Computer Science. Springer-Verlag, Berlin, July 1997.

[19] M. Kerckhove, editor. Scale-Space and Morphology in Computer Vision: Proceedings of the Third International Conference, Scale-Space 2001, Vancouver, Canada, volume 2106 of Lecture Notes in Computer Science. Springer-Verlag, Berlin, July 2001.

[20] R. Kimmel, N. Sochen, and J. Weickert, editors. Scale Space and PDE Methods in Computer Vision: Proceedings of the Fifth International Conference, Scale-Space 2005, Hofgeismar, Germany, volume 3459 of Lecture Notes in Computer Science. Springer-Verlag, Berlin, April 2005.

[21] J. J. Koenderink. The structure of images. Biological Cybernetics, 50:363-370, 1984.

[22] J. J. Koenderink and A. J. van Doorn. Receptive field families. Biological Cybernetics, 63:291-298, 1990.

[23] C. Lenglet, R. Deriche, and O. Faugeras. Inferring white matter geometry from diffusion tensor MRI: Application to connectivity mapping. In T. Pajdla and J. Matas, editors, Proceedings of the Eighth European Conference on Computer Vision (Prague, Czech Republic, May 2004), volume 30213024 of Lecture Notes in Computer Science, pages 127-140, Berlin, May 2004. Springer-Verlag.

[24] T. Lindeberg. Scale-Space Theory in Computer Vision. The Kluwer International Series in Engineering and Computer Science. Kluwer Academic Publishers, Dordrecht, The Netherlands, 1994.

[25] J. Melonakos, E. Pichon, S. Angenent, and A. Tannenbaum. Finsler active contours. IEEE Transactions on Pattern Analysis and Machine Intelligence, 30(3):412-423, 2008.

[26] C. W. Misner, K. S. Thorne, and J. A. Wheeler. Gravitation. Freeman, San Francisco, 1973.

[27] C. Moler and C. Van Loan. Nineteen dubious ways to compute the exponential of a matrix. SIAM Review, 20(4):801836, October 1978.

[28] I. Najfeld. Derivatives of the matrix exponential and their computation. Technical Report TR-33-94, Harvard Medical School, Boston, MA 02115-5718, 1994.

[29] I. Najfeld and T. F. Havel. Derivatives of the matrix exponential and their computation. Advances in Applied Mathematics, 16:321-375, 1995.

[30] M. Nielsen, L. M. J. Florack, and R. Deriche. Regularization, scale-space and edge detection filters. Journal of Mathematical Imaging and Vision, 7(4):291-307, October 1997.

[31] M. Nielsen, P. Johansen, O. F. Olsen, and J. Weickert, editors. Scale-Space Theories in Computer Vision: Proceedings of the Second International Conference, Scale-Space'99, Corfu, Greece, volume 1682 of Lecture Notes in Computer Science. Springer-Verlag, Berlin, September 1999.

[32] M. Ortiz, R. A. Radovitzky, and E. A. Repetto. The computation of the exponential and logarithmic mappings and their first and second linearizations. International Journal for $\mathrm{Nu}$ merical Methods in Engineering, 52:1431-1441, 2001.

[33] E. Özarslan and T. H. Mareci. Generalized diffusion tensor imaging and analytical relationships between diffusion tensor imaging and high angular resolution imaging. Magnetic Resonance in Medicine, 50:955-965, 2003.
[34] X. Pennec, P. Fillard, and N. Ayache. A Riemannian framework for tensor computing. International Journal of Computer Vision, 66(1):41-66, January 2006.

[35] P. Perona and J. Malik. Scale-space and edge detection using anisotropic diffusion. IEEE Transactions on Pattern Analysis and Machine Intelligence, 12(7):629-639, July 1990.

[36] E. Prados, S. Soatto, C. Lenglet, J.-P. Pons, N. Wotawa, R. Deriche, and O. Faugeras. Control theory and fast marching techniques for brain connectivity mapping. In Proceedings of the IEEE Computer Society Conference on Computer Vision and Pattern Recognition (New York, USA, June 2006), volume 1, pages 1076-1083, New York, USA, 2006. IEEE Computer Society Press.

[37] L. Schwartz. Théorie des Distributions. Publications de l'Institut Mathématique de l'Université de Strasbourg. Hermann, Paris, second edition, 1966.

[38] F. Sgallari, A. Murli, and N. Paragios, editors. Scale Space and Variational Methods in Computer Vision: Proceedings of the First International Conference, SSVM 2007, Ischia, Italy, volume 4485 of Lecture Notes in Computer Science. Springer-Verlag, Berlin, May-June 2007.

[39] M. Spivak. Differential Geometry, volume 1-5. Publish or Perish, Berkeley, 1975.

[40] A. Tikhonov and V. Y. Arseninn. Solution of Ill-Posed Problems. John Wiley \& Sons, New York, 1977.

[41] L. J. v. Vliet, I. T. Young, and P. W. Verbeek. Recursive Gaussian derivative filters. In A. K. Jain, S. Venkatesh, and B. C. Lowell, editors, Proceedings of the 14th International Conference on Pattern Recognition (Brisbane, Australia, August 16-20, 1998), volume 1, pages 509-514. IEEE Computer Society Press, 1998.

[42] J. A. Weickert. Anisotropic Diffusion in Image Processing. ECMI Series. Teubner, Stuttgart, January 1998.

[43] J. A. Weickert. Coherence-enhancing diffusion filtering. International Journal of Computer Vision, 31(2/3):111-127, April 1999.

[44] J. A. Weickert, S. Ishikawa, and A. Imiya. Linear scale-space has first been proposed in Japan. Journal of Mathematical Imaging and Vision, 10(3):237-252, May 1999.

[45] A. P. Witkin. Scale-space filtering. In Proceedings of the International Joint Conference on Artificial Intelligence, pages 1019-1022, Karlsruhe, Germany, 1983.
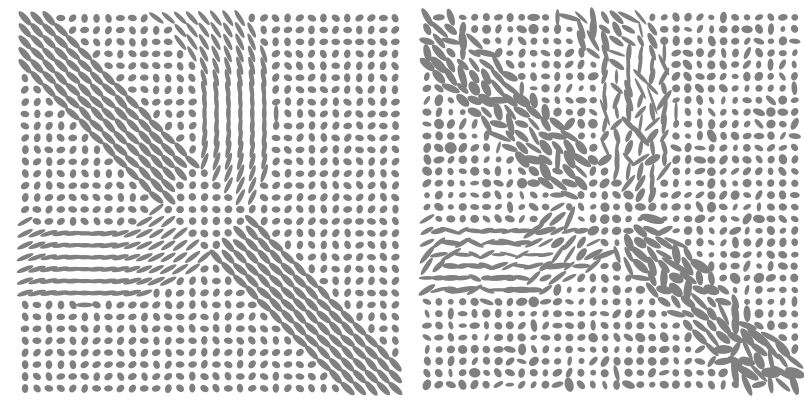

Figure 2. Two-dimensional synthetic image showing a positive symmetric tensor field unperturbed (left) and perturbed by noise (right). The second is subject to the scale space extension illustrated in Fig. 3. 


\section{A. Hessian of the Matrix Exponential Function}

Theorem 3 Let $X \in C^{\omega}\left(\mathbb{R}^{k}, \mathbb{M}_{n}\right)$. The Hessian of the matrix exponential function $\exp X \in C^{\omega}\left(\mathbb{R}^{k}, \mathbb{M}_{n}^{+}\right)$can be represented component-wise as follows:

$$
\begin{aligned}
\partial_{\mu} \partial_{\nu} & \exp (X(x))=\int_{0}^{1} \exp ((1-\sigma) X(x)) \partial_{\mu} \partial_{\nu} X(x) \exp (\sigma X(x)) d \sigma \\
& \left.+\int_{0}^{1} \int_{0}^{\sigma} \exp ((1-\sigma) X(x)) \partial_{\nu} X(x) \exp (\sigma-\rho) X(x)\right) \partial_{\mu} X(x) \exp (\rho X(x)) d \rho d \sigma \\
& +\int_{0}^{1} \int_{0}^{\sigma} \exp ((1-\sigma) X(x)) \partial_{\mu} X(x) \exp ((\sigma-\rho) X(x)) \partial_{\nu} X(x) \exp (\rho X(x)) d \rho d \sigma .
\end{aligned}
$$

Note that if $[\nabla X, X]=0$ (always the case if $n=1$, hardly ever otherwise), this expression reduces to $\partial_{\mu} \partial_{\nu} \exp (X)=$ $\left(\partial_{\mu} \partial_{\nu} X+\partial_{\mu} X \partial_{\nu} X\right) \exp (X)$, as it should.

Proof of Theorem 3. Apply the Theorem 2 twice, and perform a change of variables so as to obtain the integral expression stated in the theorem.

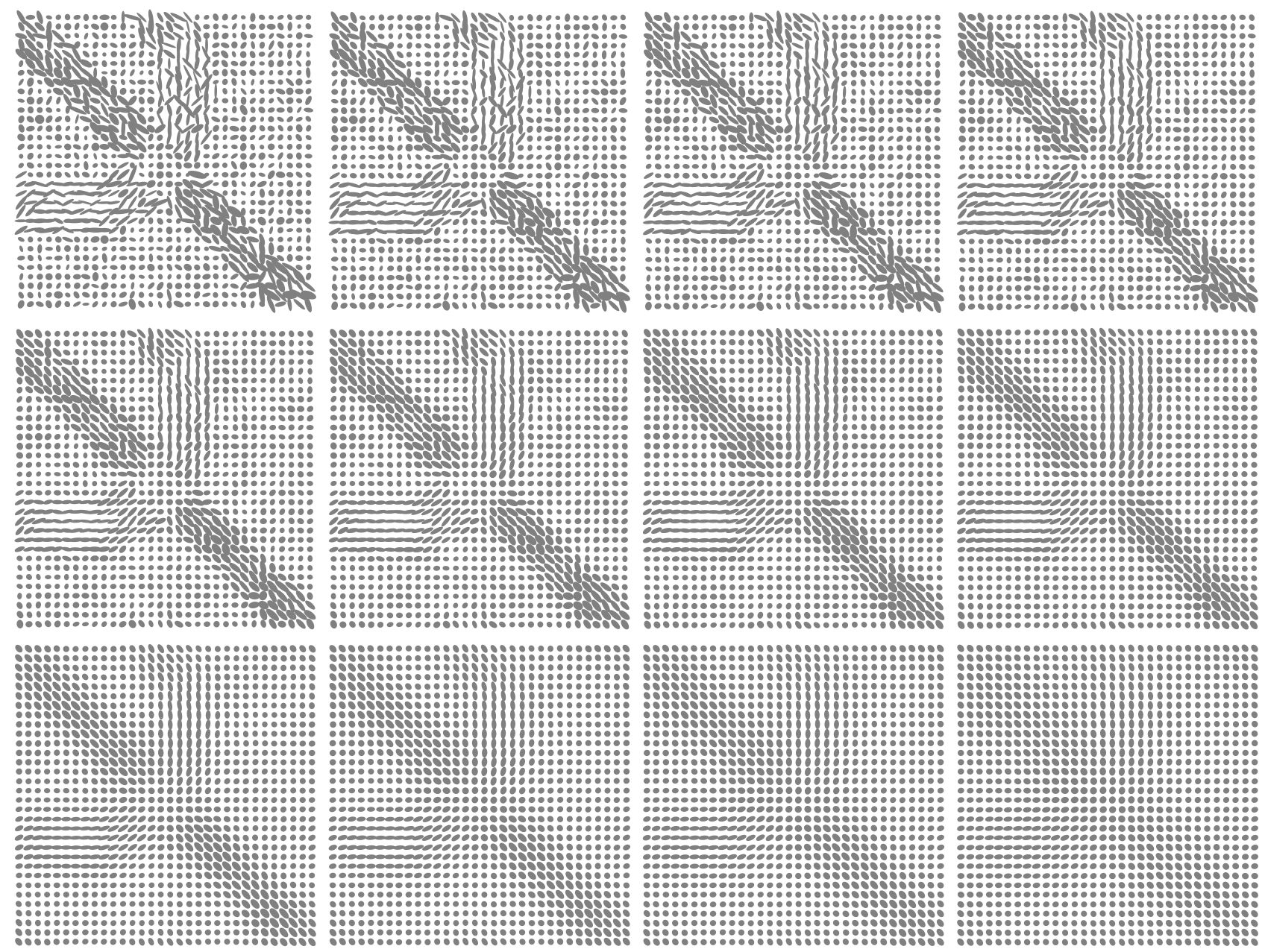

Figure 3. Illustration of Eq. (7) for twelve levels of exponentially increasing scales. The two-dimensional synthetic data are of course not very realistic, and are only provided for the sake of illustration. Qualitative behaviour and asymptotics will be similar in practice. 